preprint INRNE-TH-97/1 quant-ph/9701018)

(June, 1997: minor changes in text and references)

Accepted in Journal of Physics A

\title{
ROBERTSON INTELLIGENT STATES
}

\author{
D.A. Trifonov \\ Institute for Nuclear Research \\ 72 Tzarigradsko chaussée \\ 1784 Sofia, Bulgaria
}

\begin{abstract}
Diagonalization of uncertainty matrix and minimization of Robertson inequality for $n$ observables are considered. It is proved that for even $n$ this relation is minimized in states which are eigenstates of $n / 2$ independent complex linear combinations of the observables. In case of canonical observables this eigenvalue condition is also necessary. Such minimizing states are called Robertson intelligent states (RIS). The group related coherent states (CS) with maximal symmetry (for semisimple Lie groups) are particular case of RIS for the quadratures of Weyl generators. Explicit constructions of RIS are considered for operators of $s u(1,1), s u(2), h_{N}$ and $s p(N, R)$ algebras. Unlike the group related CS, RIS can exhibit strong squeezing of group generators. Multimode squared amplitude squeezed states are naturally introduced as $s p(N, R)$ RIS. It is shown that the uncertainty matrices for quadratures of $q$-deformed boson operators $a_{q, j}(q>0)$ and of any $k$ power of $a_{j}=a_{1, j}$ are positive definite and can be diagonalized by symplectic linear transformations.
\end{abstract}

\section{Introduction}

Canonical coherent states (CS) [1, 2] in quantum optics and quantum mechanics can be defined in three equivalent ways: 1) as eigenstates of nonhermitean boson (photon) destruction operator $a ; 2$ ) as orbit of the oscillator ground state $|0\rangle$ under the action of unitary displacement operator $D(\alpha) ; 3)$ as states which minimize the Heisenberg relation for canonical observables $q=\left(a+a^{\dagger}\right) / \sqrt{2}$ and $p=-i\left(a-a^{\dagger}\right) / \sqrt{2}$ with equal uncertainties. Correspondingly there are three ways of generalizations of canonical CS [3]. As most general up to now is considered the second one, which consists in construction orbits of a reference vectors $\left|\psi_{0}\right\rangle$ under the action of unitary operators of irreducible representations of a given Lie group [2, 3] $(D(\alpha)$ realize ray representation of Heisenberg-Weyl group $H_{1}$ ). These generalized CS are known (and should be called here) as group related CS [2].

The main aim of the present paper is to consider the third way of generalization (the intelligence way) to the case of $n$ observables and its relationship to the first two 
ones. The idea is to look for a generalized uncertainty relation for $n$ observables $X_{\mu}$, $\mu=1,2, \ldots, n$, which minimization would yield a continuous family of states such that in cases of $X_{\mu}$ being generators of a Lie group it would include the corresponding group related CS.

It turned out that the required generic uncertainty relation (UR) for $n$ observables is that of Robertson [4], eq. (11), (see also the review [5] on generalized uncertainty relations). Here we show that it is minimized in the eigenstates of $n / 2$ (for even $n$ ) independent complex linear combinations of $X_{\mu}$ or (for any $n$ ) of at least one real combination. When $X_{\mu}$ are quadrature components of Weyl generators of a semisimple Lie group [6] these minimizing states contain as subset the corresponding group related CS with symmetry [2, 3]. Thus it is Robertson relation that naturally connects the above three ways of generalization of CS on the level of $n$ observables. In case of $N$ mode electromagnetic field we get that Robertson UR (RUR) is minimized if and only if the state is an eigenstate of $N$ new destruction operators $a_{j}^{\prime}=u_{j k} a_{k}+v_{j k} a_{k}^{\dagger}$. For brevity states which minimize some uncertainty relation should be called here intelligent states (IS) (the term IS is introduced in [0] on the example of spin states which minimize Heisenberg UR). The term correlated [8] is reserved for states with nonvanishing covariances (correlations).

The first step on the first way of CS generalization was made in papers [9, 10, 11] where eigenstates of complex combinations of $a_{j}$ and $a_{j}^{\dagger}, j=1,2 \ldots, N$, were constructed and discussed $(N=2$ in [9], $N=1$ in [10], any $N$ in [11]). Later [8] it was shown that eigenstates of $u a+v a^{\dagger}$ minimize the Schrödinger UR (SUR) [12 for $q$ and $p$ (eq. (3)), the minimizing states being called correlated CS. Those CS in fact are the same [13] as canonical squeezed states in quantum optics [14]. In [15] it was proved that SUR for any two observables $X$ and $Y$ is minimized in eigenstates of their complex combination $\lambda X+i Y$, (equivalently of $u A+v A^{\dagger}, A=(X+i Y) / \sqrt{2}$, $\lambda$ and $u, v$ are complex numbers). Eigenstates of $u A+v A^{\dagger}$ can exhibit strong squeezing in $X$ and $Y$. Schrödinger IS (SIS) for the generators $K_{1}, K_{2}$ of $S U(1,1)$ were constructed in [15] and shown to combine the Barut-Girardello CS [16] and $S U(1,1)$ group related CS with symmetry [3]. The full sets of even and odd SIS for quadratures of squared boson destruction operator $a^{2}$ were constructed in the second paper of ref. [19] (see also [31, 30]). Eigenstates of $u A+v A^{\dagger}$ with real $u$ and $v$ are noncorrelated SIS, that is Heisenberg IS, and the cases of $X, Y$ being quadratures of $a a$ or of the product $a b$ of two annihilation operators were considered in papers [17].

Another purpose of the paper is to consider the diagonalization problem of uncertainty matrix, denoted here by $\sigma$. This matrix is of direct physical significance since its elements are dispersions (variances) and correlations (covariances) of ob- 
servables. $\sigma$ is important also in quantum state geometry [18. In case of canonical operators $q_{j}, p_{k}$ diagonalization of $\sigma$ was considered in [19].

The paper is organized as follows. In section 2 we briefly review Robertson relations for the uncertainty matrix $\sigma$ for $n$ observables $X_{\mu}$. In section 3 we consider the diagonalization of $\sigma$ by means of linear transformations of $X_{\mu}$. We note that in any state $\sigma$ can be diagonalized by means of orthogonal transformation. From this it follows that the spin component correlations can be eliminated by coordinate rotation. When the uncertainty matrix is positive definite (as is the case of $2 \mathrm{~N}$ quadratures of $k$ power of boson/photon annihilation operators $a_{j}$ and the case of quadratures of $q$-deformed boson operators $a_{j, q}$ for $q>0$ ) it can be diagonalized also by means of symplectic transformation. New family of trace class UR (15) is established for positive definite dispersion matrices.

In section 4 we study the minimization of $n$ dimensional RUR. In section 5 explicit examples of RIS are considered, the $s u(1,1)$ and $s u(2)$ RIS being discussed in greater detail. RIS for generators of $S U(1,1)$ in quadratic bosonic representation can exhibit linear and quadratic amplitude squeezing (even simultaneously - joint squeezing of two noncommuting observables).

\section{Robertson uncertainty inequalities}

For $n$ observables (hermitean operators) $X_{\mu}$ Robertson 《4 (see also review paper [5]) established the following two uncertainty relations for the dispersion matrix $\sigma$,

$$
\begin{gathered}
\operatorname{det} \sigma \geq \operatorname{det} C, \\
\sigma_{11} \sigma_{22} \ldots \sigma_{n n} \geq \operatorname{det} \sigma,
\end{gathered}
$$

where $\sigma_{\mu \nu}=\left\langle X_{\mu} X_{\nu}+X_{\nu} X \mu\right\rangle / 2-\left\langle X_{\mu}\right\rangle\left\langle X_{\nu}\right\rangle$ and $C$ is the antisymmetric matrix of mean commutators, $C_{\mu \nu}=-(i / 2)\left\langle\left[X_{\mu}, X_{\nu}\right]\right\rangle$. Here $\langle X\rangle$ is the mean value of $X$ in quantum state $\rho$, which is generally mixed state. For $n=2$ inequality (1) coincides with $\operatorname{SUR}\left(X_{1}=X, X_{2}=Y\right)$

$$
\Delta^{2} X \Delta^{2} Y-\sigma^{2} X Y \geq \frac{1}{4} \mid\left\langle\left.[X, Y]\right|^{2}\right.
$$

which in turn is reduced to the Heisenberg UR for $X$ and $Y$ when the covariance $\sigma_{X Y}=\langle X Y+Y X\rangle / 2-\langle X\rangle\langle Y\rangle$ is vanishing $\left(\Delta^{2} X \equiv \sigma_{X X}, \Delta^{2} Y \equiv \sigma_{X Y}\right)$.

Combining (11) and (2) one gets

$$
\sigma_{11} \sigma_{22} \ldots \sigma_{n n} \geq \operatorname{det} C
$$

which can be treated as direct extension of Heisenberg UR to the case of $n$ operators. 
The uncertainty matrix (dispersion or correlation matrix) $\sigma=\sigma(\vec{X}, \rho)$, where $\vec{X}=\left(X_{1}, X_{2}, \ldots, X_{n}\right)$, is symmetric by construction. From Robertson inequalities (11), (2) one can deduce that its determinant is always nonnegative. Indeed, the matrix of mean commutators is antisymmetric and the determinant of antisymmetric matrix is nonnegative [20]. Thereby in any state $\rho$ we have $\operatorname{det} C \geq 0 . \operatorname{det} C$ vanishes identically if the number of operators $n$ is odd.

Diagonal elements of $\sigma$ are the variances of $X_{\mu}$. The problem of reducing (squeezing) of variances of quantum observables is of importance in physics (in quantum optics [14]) of precise measurements and telecommunications. The nondiagonal elements are the covariances of $X_{\mu}$ and $X_{\nu}$ and describe $X_{\mu}-X_{\nu}$ correlations. The uncertainty matrix in pure state $\left|\psi_{0}\right\rangle$ can be used as a metric tensor in the manifold of generalized Glauber CS $D(\alpha)\left|\psi_{0}\right\rangle$ [18]. In view of these dynamical and geometrical properties of $\sigma$ it is desirable to study the problem of its diagonalization (which is equivalent to the problem of minimization of the second Robertson relation (2)). Diagonalization of $\sigma$ in the case of canonical observables $p_{j}=X_{j}, q_{j}=X_{N+j}$, $j=1,2, \ldots, N$, was recently considered in [19]: in any state it can be diagonalized by means of linear canonical transformations. In the next section we consider this problem in more general cases. The minimization of (1) for two observables $X_{1}$ and $X_{2}$ (i.e. of SUR (3)) has been shown [15] to occur in the eigenstates of their complex (in particular real) linear combinations only. In section 4 we extend this result to arbitrary $n$.

\section{Diagonalization of uncertainty matrix of $n$ observables}

In this section we consider the diagonalization of the uncertainty matrix $\sigma(\vec{X}, \rho)$ by means of linear transformations of $n$ operators $X_{\mu}$ (summation over repeated indices),

$$
X_{\mu} \rightarrow X_{\mu}^{\prime}=\lambda_{\mu \nu} X_{\nu}
$$

where $\lambda_{\mu \nu}$ are real numbers (in order $X_{\mu}^{\prime}$ to be again hermitean operators).

We first note the transformation property of $\sigma$ under transformation (5). Defining the new matrix $\sigma^{\prime}$ as $\sigma^{\prime}=\sigma\left(\vec{X}^{\prime}, \rho\right)$ we easily get

$$
\sigma^{\prime}=\Lambda \sigma \Lambda^{T}
$$

where we introduced $n$ vector $\vec{X}^{\prime}=\left(X_{1}^{\prime}, \ldots, X_{n}^{\prime}\right)$ and $n \times n$ matrix $\Lambda=\left\{\lambda_{\mu \nu}\right\}$, its transposed being denoted as $\Lambda^{T}$. Thus the two dispersion matrices are congruent via the transformation matrix $\Lambda$. We suppose that transformation (5) is invertable and set $\operatorname{det} \Lambda=1$. In matrix form eq. (5) is rewritten as $\vec{X}^{\prime}=\Lambda \vec{X}$. 
We note several general properties of $\sigma$, some of which being immediate consequences of its symmetricity and the transformation law (6). First we note the invariant quantities: a) $\operatorname{det} \sigma=\operatorname{det} \sigma^{\prime}$ for any $\Lambda \in S L(n, C)$; b) $\operatorname{Tr} \sigma^{k}=\operatorname{Tr} \sigma^{\prime k}$, $k=1,2, \ldots$, for orthogonal $\Lambda$; c) $\operatorname{Tr}(\sigma J)^{k}=\operatorname{Tr}\left(\sigma^{\prime} J\right)^{k}$ for symplectic transformations $(n=2 N)$,

$$
\Lambda J \Lambda^{T}=J, \quad J=\left(\begin{array}{ll}
0 & 1_{N} \\
-1_{N} & 0
\end{array}\right) .
$$

The last two invariants are particular cases of quite general relations $\operatorname{Tr}\left(\sigma^{\prime} g\right)^{k}=$ $\operatorname{Tr}(\sigma g)^{k}$ which hold for $\Lambda$ satisfying $\Lambda^{T} g \Lambda=g$ with any fixed matrix $g$ (in the above $g=1$ and $g=J)$.

Next we note that $\sigma$ (being symmetric) can be always diagonalized by means of orthogonal $\Lambda\left(\Lambda \Lambda^{T}=1\right)$ 20] in any state, i.e. $\sigma^{\prime}$ is diagonal for some orthogonal $\Lambda$. In case of spin (or angular momentum) operators we get from this property that spin component correlations can be considered as pure coordinate effects. An other general property of $\sigma$ is its nonnegativity, $\sigma \geq 0$. To prove this last property we diagonalize $\sigma$ by means of orthogonal matrix $\Lambda$. The new operators $X_{\mu}^{\prime}$, eq. (5), are again hermitean and therefor all the diagonal elements of the matrix $\sigma^{\prime}$ are nonnegative. Therefor $\sigma \geq 0$ in any state $\rho$.

Further properties of the uncertainty matrix can be established when the set of operators $X_{\mu}$ possess some additional properties. For example if $\sigma$ is positive definite, $\sigma>0$, then it can be diagonalized by means of symplectic $\Lambda$ [21]. Therefor it is important to know when the uncertainty matrix is strictly positive. The value of $\operatorname{det} C \geq 0$ turned out to play important role. Note that $\operatorname{det} \sigma>0$ stems from $\sigma>0$ and $\operatorname{det} \sigma=0$ means that $\sigma$ is not strictly positive.

Proposition 1. $\operatorname{det} \sigma(\vec{X}, \rho)=0$ in pure states $\rho=|\psi\rangle\langle\psi|$ if and only if $|\psi\rangle$ is an eigenstate of a real combination $\lambda_{\nu} X_{\nu}$ of $X_{\nu}$.

Proof. a) Necessity. Let $\operatorname{det} \sigma(\vec{X}, \rho)=0$. Then orthogonal $\Lambda$ exists such that $\sigma^{\prime}$ is diagonal. We have $0=\operatorname{det} \sigma=\operatorname{det} \sigma^{\prime}=\sigma_{11}^{\prime} \sigma_{22}^{\prime} \ldots \sigma_{n n}^{\prime}$, wherefrom at least for one $\nu$ one has $\sigma_{\nu \nu}^{\prime}=0$. The latter is possible in pure states $\rho=|\psi\rangle\langle\psi|$ if and only if $X_{\nu}^{\prime}|\psi\rangle=x_{\nu}^{\prime}|\psi\rangle$. b) Sufficiency. Let $(\vec{\lambda} \vec{X})|\psi\rangle=x^{\prime}|\psi\rangle, \vec{\lambda} \vec{X} \equiv \lambda_{\nu} X_{\nu}$. Then we can always construct nondegenerate matrix $\Lambda$ with first row $\left(\lambda_{1}, \lambda_{2}, \ldots, \lambda_{n}\right)$ and consider the uncertainty matrix $\sigma^{\prime}=\sigma(\Lambda \vec{X} ; \psi)$. This $\sigma^{\prime}$ is with vanishing determinant since the first column of it is zero (as a consequence of $(\vec{\lambda} \vec{X})|\psi\rangle=x^{\prime}|\psi\rangle$ ). But $0=\operatorname{det} \sigma^{\prime}=(\operatorname{det} \Lambda)^{2} \operatorname{det} \sigma$, therefor $\operatorname{det} \sigma=0$.

In view of this proposition and eq. (1) one has the

Corollary 1: If $\operatorname{det} C(\vec{X}, \psi)>0$ then $|\psi\rangle$ can't be normalizable eigenstate of 
any real combination $\lambda_{\nu} X_{\nu}$.

If $\operatorname{det} C(\vec{X} ; \psi)>0$ in any state then neither $X_{\mu}$ nor any real combination $\lambda_{\nu} X_{\nu}$ can be diagonalized in Hilbert space of states $\mathcal{H}$, that is the spectrum of $X_{\mu}$ and $\lambda_{\nu} X_{\nu}$ are continuous. Here is a class of $2 N$ operators for which $\operatorname{det} C>0$ and therefor $\sigma$ is positive in any state.

Proposition 2. If $X_{\mu}, \mu=1,2, \ldots, 2 N$ obey the commutation relations

$$
\left[X_{j}, X_{n+k}\right]=\delta_{j k}\left[X_{j}, X_{n+j}\right], \quad\left[X_{j}, X_{k}\right]=0=\left[X_{n+j}, X_{n+k}\right]
$$

where $-i\left[X_{j}, X_{n+j}\right]$ are positive definite operators, then $\operatorname{det} C(\vec{X} ; \rho)>0$ and the uncertainty matrix $\sigma(\vec{X} ; \rho)$ is positive definite.

Proof. By direct calculations we get

$$
\operatorname{det} C=\left(\frac{1}{2}\right)^{2 N}\left\langle(-i)\left[X_{1}, Y_{1}\right]\right\rangle^{2} \cdot\left\langle(-i)\left[X_{2}, Y_{2}\right]\right\rangle^{2} \ldots\left\langle(-i)\left[X_{N}, Y_{N}\right]\right\rangle^{2},
$$

where $Y_{j} \equiv X_{N+j}, j=1,2, \ldots, N$. Since every factor in (9) is positive one has $\operatorname{det} C>0$. From corollary 1 and the diagonalization of $\sigma$ by orthogonal $\Lambda$ we derive that $\operatorname{det} C>0$ is a sufficient condition for $\sigma$ to be positive definite. End of proof.

We can point out a family of boson system (e.g. $N$ mode electromagnetic field) observables which obey the commutation relations (\&). Those are the quadrature components of power $k$ of photon (boson) destruction operators $a_{j}$, defined here as

$$
X_{j}^{(k)}=\frac{1}{\sqrt{2 k}}\left(a_{j}^{k}+a_{j}^{k \dagger}\right), \quad X_{N+j}^{(k)}=\frac{-i}{\sqrt{2 k}}\left(a_{j}^{k}-a_{j}^{k \dagger}\right) \equiv Y_{j} .
$$

The relations (8) and the positivity of $-i\left[X_{j}^{(k)}, Y_{j}^{(k)}\right]=(1 / k)\left[a^{k}, a^{k \dagger}\right]$ can be checked by direct calculations. As a result the quadrature components of $a^{k}$ are continuous observables, their uncertainty matrix is positive definite and can be diagonalized by means of symplectic $\Lambda$. For $k=1$ operators (10) are the canonical pairs $q_{j}, p_{j}$, therefor their uncertainty matrix can be diagonalized by means of linear canonical transformations, corresponding to symplectic $\Lambda$. The procedure for diagonalization of positive definite matrix by means of symplectic $\Lambda$ is described in [21] and in the first paper of ref. [19]. Canonical transformations with time dependent $\Lambda(t)$ can be used to diagonalize any quadratic Hamiltonian. For oscillator with varying mass and/or frequency this is done by Seleznyova [22].

Positive definite uncertainty matrices exist also in q-deformed boson systems. $q$-deformed oscillator is introduced in [23]. The deformed lowering and raising operators $a_{q}$ and $a_{q}^{\dagger}$ obey the commutation relation

$$
\left[a_{q}, a_{q}^{\dagger}\right]=\left[N_{q}+1\right]-\left[N_{q}\right], \quad[N] \equiv \frac{q^{N}-q^{-N}}{q-q^{-1}}
$$


where $N_{q}$ is a number operator which eigenstates are $|n\rangle_{q}=([n] !)^{-1 / 2} a_{q}^{\dagger n}|0\rangle_{q}$ : $N_{q}|n\rangle_{q}=n|n\rangle_{q}, \quad a_{q}|0\rangle_{q}=0,[n] !=[n][n-1] \ldots[1]$. At $q=1 \quad a_{q}, a_{q}^{\dagger}$ coincide with ordinary boson operators $a, a^{\dagger}$. Now we note that the commutator $\left[a_{q}, a_{q}^{\dagger}\right]$ is positive definite for $q>0$ as one can easily verify, using (11). From the commutation relations for $n q$-deformed oscillators [24]

$$
\begin{array}{r}
{\left[a_{q, j}, a_{q, k}\right]=0, \quad\left[a_{q, j}, a_{q, k}^{\dagger}\right]=\delta_{j k}\left[a_{q, j}, a_{q, j}^{\dagger}\right]} \\
{\left[N_{q, j}, a_{q, k}\right]=-\delta_{j k} a_{q, k}, \quad\left[N_{q, j}, a_{q, k}^{\dagger}\right]=\delta_{j k} a_{q, j}^{\dagger}}
\end{array}
$$

it follows that the set of quadrature components of $a_{q, j}$ obey the requirements of proposition 2 for $q>0$. Therefor the uncertainty matrix $\sigma\left(\vec{X}_{q} ; \rho\right)$ is positive definite in any state for $q>0$.

For positive definite uncertainty matrix of $2 N$ observables satisfying (8) one can establish a set of new uncertainty relations. In this purpose consider the invariant quantities $\operatorname{Tr}(i \sigma J)^{2 k}, k=1,2, \ldots$, . Let $\sigma^{\prime}$ be diagonal matrix which is symplectically congruent to $\sigma$. Then we have

$$
\operatorname{Tr}(i \sigma J)^{2 k}=\operatorname{Tr}\left(i \sigma^{\prime} J\right)^{2 k}=2 \sum_{j}^{N}\left[\sigma_{j, j}^{\prime} \sigma_{N+j, N+j}^{\prime}\right]^{k}
$$

In view of $\sigma>0$ every term $\sigma_{j, j}^{\prime} \sigma_{N+j, N+j}^{\prime}$ in (13) is nonvanishing and positive. We can apply the Heisenberg relation for $\sigma_{j, j}^{\prime} \sigma_{N+j, N+j}^{\prime}$ and write the set of inequalities

$$
\operatorname{Tr}(i \sigma J)^{2 k} \geq 2 \sum_{j}^{N}\left|\frac{1}{2}\left\langle\left[X_{j}^{\prime}, X_{N+j}^{\prime}\right]\right\rangle\right|^{k} .
$$

In the above $X_{\mu}^{\prime}=\Lambda_{\mu \nu}(\rho) X_{\nu}$ and $\Lambda(\rho)$ is the diagonalizing symplectic matrix for the state $\rho$. For every state we can in principle find the minimal value $c_{0}^{2}(\rho)$ of the $N$ terms $\left|\left\langle\left[X_{j}^{\prime}, X_{N+j}^{\prime}\right]\right\rangle\right|$ and therefor rewrite (14) in a more compact form

$$
\operatorname{Tr}(i \sigma J)^{2 k} \geq \frac{N}{2^{2 k-1}} c_{0}^{2 k}, \quad k=1,2, \ldots
$$

In particular case of canonical variables $X_{j}=p_{j}, X_{N+j}=q_{j}$ in any state the products $\sigma_{j, j}^{\prime} \sigma_{N+j, N+j}^{\prime}$ are greater or equal to $1 / 4$ (this is the value of $\sigma_{j, j}^{\prime} \sigma_{N+j, N+j}^{\prime}$ in Glauber CS for mode $j, \hbar=1$ ), that is $c_{0}^{2} \geq 1$. Thus for canonical variables the above UR read $\left(\vec{Q}=\left(p_{1}, \ldots, p_{N}, q_{1}, \ldots, q_{N}\right)\right)$

$$
\operatorname{Tr}[i \sigma(\vec{Q}, \rho) J]^{2 k} \geq \frac{N}{2^{2 k-1}} .
$$

The latter inequalities for the case of $\sigma(\vec{Q}, \rho)$ (apart from the factor $i$ ) were recently obtained by Sudarshan, Chiu and Bhamathi [19]. For $N=1$ and $k=1$ the inequality (16) recovers the Schrödinger relation (3). 
The above considered diagonalization of uncertainty matrix of $n$ hermitean operators by means of transformations of operators $X_{\mu} \rightarrow X_{\mu}^{\prime}$ should be referred here as first kind diagonalization. The state $\rho$ here is kept the same. This diagonalization is always possible as we have shown. But it is of interest also to know when $\sigma$ can be diagonalized by state transformation, keeping observables the same. That is for given $X_{\mu}$ and state $\rho$ to find new state $\rho^{\prime}$ so that the new matrix $\sigma^{\prime \prime} \equiv \sigma\left(\vec{X}, \rho^{\prime}\right)$ be diagonal. We shall call this second kind diagonalization. Evidently both diagonalizations coincide (i.e. $\sigma^{\prime}=\sigma^{\prime \prime}$ ) when the transformation (5) is generated by some unitary operator $U(\Lambda)$,

$$
X_{\mu}^{\prime}=\lambda_{\mu \nu} X_{\nu}=U^{\dagger}(\Lambda) X_{\mu} U(\Lambda)
$$

Such is the case e.g. of uncertainty matrix $\sigma(\vec{Q}, \rho)$ of canonical operators $p_{j} \equiv Q_{j}$ and $q_{j} \equiv Q_{N+j}$ when the diagonalizing $\Lambda$ is symplectic. Then $U(\Lambda)$ is a representation of the group $S p(N, R)$ [6] (more precisely of $M p(N, R)=\overline{S p(N, R)}$ ) and thus any pure or mixed canonical correlated states is unitary equivalent to noncorrelated state. In case of $N=1$ we have an extra diagonalizing property: in view of the fact that the squared boson operators $a^{2}, a^{\dagger 2}, a^{\dagger} a$ close the $s u(1,1)$ algebra, eq. (52),$(s u(1,1) \sim s p(1, R))$ we get that in the one mode field case the quadratic amplitude dispersion matrix is also diagonalizable by unitary $M p(1, R)$ state transformation. The property (17) occurs also in the cases when $X_{\mu}$ close orthogonal algebra $s o(n, R)$. Then the diagonalizing orthogonal transformation (5) is generated by unitary $U(\Lambda) \in S O(n, R)$. On the example of $s o(3, R) \sim s u(2)$ this means (recall that if $J_{k}$ are spin operators, $\left[J_{k}, J_{j}\right]=i \hbar \epsilon_{k j l} J_{l}$, and $\Lambda$ is orthogonal then $\left[J_{k}^{\prime}, J_{j}^{\prime}\right]=i \hbar \epsilon_{k j l} J_{l}^{\prime}$ ) that spin component correlations (covariances) in any state can always be eliminated by means of coordinate rotation (first kind diagonalization) and by state transformation with unitary operator $U(\Lambda)$ (second kind diagonalization). In other words spin component correlation is pure coordinate effect and any spin correlated state is unitary equivalent to a noncorrelated one.

\section{Minimization of Robertson uncertainty inequality $\operatorname{det} \sigma \geq \operatorname{det} C$}

One general sufficient condition for minimization of Robertson inequality (1) for arbitrary observables $X_{\mu}, \mu=1,2, \ldots, n$, follows from the proposition 1: the equality in (11) holds in the eigenstates of at least one of $X_{\mu}$ since in such case both matrices $\sigma$ and $C$ have at least one vanishing column and then $\operatorname{det} \sigma=\operatorname{det} C=0$. In view of the fact that $\sigma$ can be always digonalized by means of orthogonal $\Lambda$ (second 
immediate property in section 2) the minimization of both Robertson relations for any $n$ occurs also in the eigenstates of some of $X_{\mu}^{\prime}=\lambda_{\mu \nu} X_{\nu}$.

In case of odd $n$ the above sufficient condition for minimization of (1) is also a necessary one: The inequality (11) is minimized in a state $|\psi\rangle$ if and only if $|\psi\rangle$ is eigenstate of a real combination $\lambda_{\nu} X_{\nu}$ of observables $X_{\nu}$. The proof follows from the proposition 1 and the property of determinant of antisymmetric matrices of odd dimension: for odd $n \operatorname{det} C$ of antisymmetric matrix $C$ is vanishing identically in any state.

$\operatorname{det} C$ can be greater than 0 for even $n$ only. For even number of operators $X_{\mu}$ we establish the following sufficient condition.

Proposition 3. The equality in the RUR (1) for $2 N$ hermitean operators $X_{\mu}$ holds in the eigenstates $|\psi\rangle$ of $N$ independent complex linear combinations of $X_{\mu}$.

Proof. Let $X_{\mu}^{\prime}=\lambda_{\mu \nu} X_{\nu} \equiv X_{\mu}^{\prime}(\Lambda)$ be some linear transformation which preserves the hermiticity, i.e. $\lambda_{\mu \nu}$ are real parameters. We introduce $N$ nonhermitean operators $A_{j}=X_{j}+i X_{N+j}$ and construct $N$ independent complex combinations of all $X_{\nu}$ in the form ,

$$
A_{j}^{\prime}=X_{j}^{\prime}+i X_{N+j}^{\prime}=u_{j k} A_{k}+v_{j k} A_{k}^{\dagger}
$$

where $u_{j k}$ and $v_{j k}$ are new complex parameters which are simply expressed in terms of $\lambda_{\mu \nu}(j, k=1,2, \ldots, N)$. Let now $|\psi\rangle$ be eigenstate of all $A_{j}^{\prime}$,

$$
A_{j}^{\prime}|\psi\rangle=z_{j}|\psi\rangle, \quad j=1,2, \ldots, N
$$

$z_{j}$ being the eigenvalue. It is natural to denote the solutions of (19) as $|\vec{z}, u, v\rangle$ or equivalently as $|\vec{z}, \Lambda\rangle$, where $u, v$ are $N \times N$ matrices and $\Lambda$ is $2 N \times 2 N$.

The scheme of the proof is to express both matrices $\sigma(\vec{X}, \psi)$ and $C(\vec{X}, \psi)$ in terms of matrices $\sigma\left(\overrightarrow{B^{\prime}}, \psi\right)$ and $C\left(\overrightarrow{B^{\prime}}, \psi\right)$ and to compare their determinants. Here $\vec{B}=\left(A_{1}, A_{2}, \ldots, A_{N}, A_{1}^{\dagger}, A_{2}^{\dagger}, \ldots, A_{N}^{\dagger}\right) \equiv\left(\vec{A}, \overrightarrow{A^{\dagger}}\right)$ and $\overrightarrow{B^{\prime}}=\left(\overrightarrow{A^{\prime}}, \overrightarrow{A^{\prime \dagger}}\right)$. First we relate $\vec{X}$ to $\vec{B}$

$$
\vec{X}=b \vec{B}, \quad b=\frac{1}{2}\left(\begin{array}{ll}
1_{N} & 1_{N} \\
i 1_{N} & -i 1_{N}
\end{array}\right),
$$

where $1_{N}$ is $N \times N$ unit matrix. We introduce $2 N \times 2 N$ transformation matrix $V$, which relates $\vec{B}$ and $\vec{B}^{\prime}$,

$$
\vec{B}^{\prime}=V \vec{B}, \quad V=\left(\begin{array}{ll}
u & v \\
v^{*} & u^{*}
\end{array}\right),
$$

where $u$ and $v$ are $N \times N$ matrices of the transformation (18). We consider the new operators $A_{j}^{\prime}$ independent (as well as the old ones $A_{j}$ ), therefor matrix $V$ is supposed 
to be invertable, that is $\operatorname{det} V \neq 0$. Using the above two linear transformation and the definition of $\sigma$ we get

$$
\sigma(\vec{X}, \psi)=b V^{-1} \sigma\left(\overrightarrow{A^{\prime}}, \psi\right)\left(V^{-1}\right)^{T} b^{T}
$$

and similarly

$$
C(\vec{X}, \psi)=b V^{-1} C\left(\vec{B}^{\prime}, \psi\right)\left(V^{-1}\right)^{T} b^{T} .
$$

Next, using the eigenvalue eqs. (19) we can prove the equality

$$
\operatorname{det} \sigma\left(\overrightarrow{B^{\prime}}, \psi\right)=\operatorname{det} C\left(\overrightarrow{B^{\prime}}, \psi\right)
$$

which in view of (22) and (23) (and non degenaracy of $b$ and $V$, $\operatorname{det} b=(-i / 2)^{N}$ ) leads to the desired equality in the RUR (11),

$$
\operatorname{det} \sigma(\vec{X}, \psi)=\operatorname{det} C(\vec{X}, \psi)
$$

The proof of auxiliary equality (24) can be carried out by direct calculations: one has

$$
\begin{aligned}
\sigma_{j k}\left(\overrightarrow{B^{\prime}}, \psi\right) & =0=C_{j k}\left(\overrightarrow{B^{\prime}}, \psi\right), \\
\sigma_{N+j, N+k}\left(\overrightarrow{B^{\prime}}, \psi\right) & =0=C_{N+j, N+k}\left(\overrightarrow{B^{\prime}}, \psi\right), \\
\sigma_{j, N+k}\left(\overrightarrow{B^{\prime}}, \psi\right) & =-i C_{j, N+k}\left(\overrightarrow{B^{\prime}}, \psi\right), \\
\sigma_{N+j, k}\left(\overrightarrow{B^{\prime}}, \psi\right) & =i C_{N+j, k}\left(\overrightarrow{B^{\prime}}, \psi\right),
\end{aligned}
$$

which manifestly ensure (24). Thus the states which satisfy eq. (19) minimize the inequality (1).

States which minimize RUR (11) for observables $\left(X_{1}, X_{2}, \ldots, X_{n}\right) \equiv \vec{X}$ should be called Robertson intelligent states for $\vec{X}$ (briefly $\vec{X}$-RIS). Equivalent terms could be Robertson minimum uncertainty states or Robertson correlated states, following for example papers [5, 8, 13. However we reserve the term correlated for states with nonvanishing correlations (covariances) only. In case of even $n$ in view of (19) and (18) RIS should be denoted as $|\vec{z}, u, v\rangle$ or $|\vec{z}, \Lambda\rangle$. For $n=2$ the relation (1) coincides with the Schrödinger one, eq. (3), and RIS are in fact SIS. For two observables the condition (19) is necessary and sufficient [15] to get equality in SUR.

Following the analogy to the known case of canonical observables $p_{j}$ and $q_{j}$ one can introduce squeeze operator [3, 14, 33] for arbitrary observables (generalized squeeze operator) $S(u, v)$ as operator which is a map from noncorrelated RIS with equal uncertainties for all pairs $X_{j}$ and $Y_{j}=X_{N+j}$ (those RIS minimize Heisenberg relation for $2 N$ operators (4)) to correlated RIS (RIS with nonvanishing covariances 
and nonequal variances). Noncorrelated RIS with equal uncertainties for $X_{j}$ and $Y_{j}$ are obtained when $u_{j k}=\delta_{j k}$ and $v_{j k}=0$ in $|\vec{z}, u, v\rangle$.

$$
S(u, v): \quad|\vec{z}, u, v\rangle=S(u, v)|\vec{z}\rangle
$$

where $|\vec{z}\rangle=|\vec{z}, u=1, v=0\rangle .|\vec{z}\rangle$ are eigenstates of all $A_{j}, j=1,2, \ldots, N$. For two arbitrary observables the operator $S(u, v)$ was introduced in [15]. This definition is of importance for generation of RIS $|\vec{z}, u, v\rangle$ from $|\vec{z}\rangle$ when the states $|\vec{z}\rangle$ are known and available. IS $|z\rangle$ with equal uncertainty for two observables $X, Y$ are constructed, in different notations, in a number of cases [1, 8, 16, 25, 26, 27]. It is interesting to note that for certain systems the squeeze operator $S(u, v)$ may exist as an isometric (not unitary) operator. Such is the case of $S(u, v)$ for the quadratures of squared boson annihilation operator $a^{2}$, considered in [31]. If $S$ is isometric only then its generator $H$ (defined by $S=\exp (i H)$ ) is a symmetric (not hermitean = selfadjoint) operator and can be considered as generalized observable 28]. In such cases representing $S=\exp (i t H)$ ( $t$ being real parameter, the time) we see from (27) that RIS (for $n=2$ in fact SIS) $|z, u, v\rangle$ can be generated from states with equal uncertainties $|z\rangle$ in a process of nonunitary evolution governed by symmetric Hamiltonian $H$. Symmetric but not selfadjoint is e.g. the particle momentum on a half line and the Hamiltonian of a particle with different mass parameters in $X, Y$ and $Z$ directions (moving in a crystal) 28.

Now a natural question of existence of RIS arises. We have a positive answer to this question for a broad class of observables $X_{\mu}$ : RIS exist for the operators of hermitean representations of semisimple Lie algebras in Hilbert space $\mathcal{H}$ and for representations of solvable algebras $L$ in finite dimensional $\mathcal{H}$. RIS may exist for infinite dimensional representations of certain solvable algebras. The existence of RIS for any finite dimensional representation of a solvable Lie algebra $L$ stems from the theorem 29] that any such representation possess at least one weight (i.e. a vector exist, which is eigenvector of all elements of $L$ ).

\section{Examples of RIS}

\subsection{RIS for Semisimple Lie algebras}

First we note that for any Lie group $G$ the group related CS [2, 3] $|\psi(g)\rangle=U(g)\left|\psi_{0}\right\rangle$ with $\left|\psi_{0}\right\rangle$ being eigenvector of at least one generator $X_{\mu}$ (these are CS with symmetry) universally are RIS for the generators of $G$. Indeed, $U(g)\left|\psi_{0}\right\rangle$ is evidently eigenstate of hermitean operator $U(g) X_{\mu} U^{\dagger}(g)(U(g)$ is unitary representation of $G)$.

Then we can apply the proposition 1 and get $\operatorname{det} \sigma(\vec{X} ; \psi(g))=0$. Here $\operatorname{det} C$ also vanishes identically with respect to $g \in G$, i.e. $\operatorname{det} \sigma(\vec{X} ; \psi(g))=\operatorname{det} C(\vec{X} ; \psi(g))=0$. 
If $\mathrm{G}$ is semisimple then hermitean generators $H_{l}$ from Cartan subalgebra always have normalizable eigenvectors $\left|\psi_{0}\right\rangle$ [6]. Therefor CS $|\psi(g)\rangle$ with these $\left|\psi_{0}\right\rangle$ as reference vector are RIS for all group generators ( with the trivial minimization: $\operatorname{det} \sigma=\operatorname{det} C=0$ identically with respect to $g \in G$ ).

We shall prove now that CS $|\psi(g)\rangle$ with maximal symmetry are RIS for the quadrature components of Weyl lowering operators $E_{-k}$ with the property $\operatorname{det} \sigma \geq$ 0 . The proof consists in application of proposition 3. The number of quadrature components $X_{k}, Y_{k}$ of all $E_{-k}$ is even, denoted by $2 n_{w}$, where $n_{w}$ is the number of Weyl operators $E_{-k}: E_{-k}=X_{k}-i X_{n_{w}+k} \equiv X_{k}-i Y_{k}, k=1,2, \ldots, n_{w}$. We shall prove that the eq. (19) ( the sufficient condition for RIS) is satisfied by CS $|\psi(g)\rangle$. As operators $A_{j}$ we take here $E_{-k}$ and as $A_{j}^{\prime}$ we have to take linear combinations of Weyl lowering and raising operators $u_{j k} E_{-k}+v_{j k} E_{k}, j, k=1,2, \ldots, n_{w}$ and then consider the eigenvalue equation

$$
\left(u_{j k} E_{-k}+v_{j k} E_{k}\right)|\vec{z}, u, v\rangle=z_{j}|\vec{z}, u, v\rangle
$$

Consider the action of $u_{j k} E_{-k}+v_{j k} E_{k}$ on the state $|\psi(g)\rangle$. One has (summation over repeated indices, $E_{k}=E_{-k}^{\dagger}, H_{l}=H_{l}^{\dagger}$ ).

$$
\begin{array}{r}
\left(u_{j k} E_{-k}+v_{j k} E_{k}\right)|\psi(g)\rangle=\left(u_{j k} E_{-k}+v_{j k} E_{k}\right) U(g)\left|\psi_{0}\right\rangle \\
=U(g) U^{-1}(g)\left(u_{j k} E_{-k}+v_{j k} E_{k}\right) U(g)\left|\psi_{0}\right\rangle \\
=U(g)\left[\left(u_{j k} \tilde{u}_{k i}+v_{j k} \tilde{v}_{k i}^{*}\right) E_{-i}+\left(u_{j k} \tilde{v}_{k i}+v_{j k} \tilde{u}_{k i}^{*}\right) E_{i}\right. \\
\left.+\left(u_{j k} \tilde{w}_{k l}+v_{j k} \tilde{w}_{k l}\right) H_{l}\right]\left|\psi_{0}\right\rangle .
\end{array}
$$

In the above we have applied the $\mathrm{BCH}$ formula to the transformations $U^{-1} E_{k} U$ $\left(k, j, i=1,2, \ldots, n_{w}, \quad l, m=1,2, \ldots, n_{c}, n_{c}\right.$ being the dimension of Cartan subalgebra)

$$
U^{-1}(g) E_{-k} U(g)=\tilde{u}_{k i} E_{-i}+\tilde{v}_{k i} E_{i}+\tilde{w}_{k l} H_{l} .
$$

Taking into account that $E_{-i}\left|\psi_{0}\right\rangle=0$ and $H_{l}\left|\psi_{0}\right\rangle=h_{l}\left|\psi_{0}\right\rangle$ we see that $|\psi(g)\rangle$ should be an eigenstate of all $A_{j}^{\prime}$ if the $n_{w} \times n_{w}$ matrices $u, v, \tilde{u}$ and $\tilde{v}$ satisfy the equation

$$
u \tilde{v}+v \tilde{u}^{*}=0
$$

In the last equation $\tilde{u}=\tilde{u}(g)$ and $\tilde{v}=\tilde{v}(g)$ should be treated as known for a given Lie group representation $U(g)$. Moreover the matrix $\tilde{u}$ is non degenerate. Therefor we always can solve the eq. (31], $v=-u \tilde{v}(g) \tilde{u}^{*-1}(g)$ and get $|\psi(g)\rangle$ as eigenstate of $A_{j}^{\prime}=u_{j k} E_{-k}+v_{j k} E_{k}$,

$$
\left(u_{j k} E_{-k}+v_{j k} E_{k}\right)|\psi(g)\rangle=z_{j}|\psi(g)\rangle
$$


with eigenvalues $z_{j}=\left(u_{j k} \tilde{w}_{k l}+v_{j k} \tilde{w}_{k l}\right) h_{l}$. In view of (32) the group related CS with maximal symmetry $|\psi(g)\rangle$ can be parametrized as RIS for $2 n_{w}$ components of Weyl generators: $|\psi(g)\rangle=|\vec{z}, u, v\rangle$ where $u, v$ are $n_{w} \times n_{w}$ matrices.

Thus we have demonstrated that states from unitary (in particular unitary and irreducible) orbits of exstremal weight vectors of semisimple Lie algebras are RIS for all basis operators $X_{\mu}$ and for the quadratures $X_{k}, Y_{k}=X_{n_{w}+k}$ of Weyl operators $E_{-k}$ as well. As far as we know this intelligence property of the group related CS wasn't noted so far in the literature.

We underline that RIS for quadrature components of Weyl generators $E_{-k}$ are more general than the group related CS with maximal symmetry: states $|\psi(g)\rangle$ are only a part of the set of solutions of eigenvalue eq. (28), corresponding to the constrain (31) on the parameters $u_{j k}$ and $v_{j k}$. On the example of $s u(1,1)$ and $s u(2)$ $\left(n_{w}=1, n_{c}=1\right)$ this was analyzed by explicit constructions of SIS $|z, u, v ; k\rangle$ in ref. 15.

It is worth noting that the propositions 1 and 3 can be applied to any subset of the operators of a given Lie algebra $L$. Therefor it makes sense to consider the eigenvalue problem for general element of the complexified algebra $L^{C}$,

$$
\left(\beta_{\nu} X_{\nu}\right)|\psi\rangle=z|\psi\rangle
$$

where $X_{\nu}(\nu=1, \ldots, n)$ are basis operators of $L$ and $\beta_{\nu}$ are complex parameters. Taking specific constrains on the complex parameters $\beta_{\nu}$ one can get various subset of RIS for less than $n$ algebra operators, in particular various $X_{j}-Y_{k}$ SIS. The property of group related CS to be part of the set of eigenstates of complex linear combinations of all algebra operators was noted in 30, 31. States that satisfy (33) could be called algebraic CS [31] or algebra eigenstates [30].

\subsection{Explicit solutions for $s u(1,1)$ and $s u(2)$ RIS}

Consider first $s u(1,1)$ case. The basis elements of $s u(1,1)$ are three operators $K_{\mu}$, $\mu=1,2,3$, which obey the relations

$$
\left[K_{1}, K_{2}\right]=-i K_{3}, \quad\left[K_{2}, K_{3}\right]=i K_{1}, \quad\left[K_{3}, K_{1}\right]=i K_{2}
$$

The Casimir operator is $C_{2}=K_{3}^{2}-K_{2}^{2}-K_{1}^{2}=k(k-1)$ and Weyl lowering and raising operators are $K_{\mp}=K_{1} \mp i K_{2}$. According to the previous discussion RIS for all three algebra operators and for any pair $K_{j}-K_{k}$ are contained in the set of eigenstates of general element of the algebra. Therefor one has to consider the eigenvalue equation for the general element of $s u^{C}(1,1)$,

$$
\left(u K_{-}+v K_{+}+w K_{3}\right)|z, u, v, w ; k\rangle=z|z, u, v, w ; k\rangle
$$


where $u, v, w$ are complex parameters, simply related to $\beta_{\nu}$ introduced in (33). This equation can be solved [31, 30] using the Barut-Girardello CS representation (BG representation) [16] or the $S U(1,1)$ group related CS representation [2, 3]). The solution can be carried out for $s u(1,1)$ representations with Bargman index $k=1 / 4,3 / 4$ and for the discrete series $k=1 / 2,1,3 / 2, \ldots$ (patricular cases of $v=0=w$ and $w=0$ were solved in [16, 15]. The Barut-Girardello CS (BG CS) $|\eta ; k\rangle$ are eigenstates of $K_{-}: K_{-}|\eta ; k\rangle=\eta|\eta ; k\rangle$. In this representation

$$
K_{+}=\eta, \quad K_{-}=2 k \frac{d}{d \eta}+\eta \frac{d^{2}}{d \eta^{2}}, \quad K_{3}=k+\eta \frac{d}{d \eta}
$$

and states $|\psi\rangle$ are represented by analytic functions $\Phi(\eta)$ which up to a certain common factor $f(|\eta|)$ are proportional to $\left\langle k ; \eta^{*} \mid \psi\right\rangle$. Orthonormalized eigenstates $|m ; k\rangle$ of $K_{3}$ are represented by monomials $\eta^{m}[\Gamma(k) /(m ! \Gamma(m+k))]^{1 / 2}$. For $u \neq 0$ the required analytic solution of (35) is 31.

$$
\Phi_{z}(\eta ; u, v, w)=N(z, u, v, w) \exp (c \eta) M\left(a, b, c_{1} \eta\right)
$$

where $N(z, u, v, w)$ is a normalization constant, $M(a, b, \eta)$ is the Kummer function (confluent hypergeometric function ${ }_{1} F_{1}(a, b ; \eta)$ ) [32], parameters $a, b, c$ and $c_{1}$ are

$$
\begin{array}{r}
a=k+\frac{z}{\sqrt{w^{2}-4 u v}}, \quad b=2 k, \\
c=-\frac{1}{2 u}\left(w+\sqrt{w^{2}-4 u v}\right), \quad c_{1}=\frac{1}{u} \sqrt{w^{2}-4 u v},
\end{array}
$$

and the normalizability conditions take the form

$$
\frac{1}{2|u|}\left|w-\sqrt{w^{2}-4 u v}\right|<1, \quad \text { or } \quad \frac{1}{2|u|}\left|w+\sqrt{w^{2}-4 u v}\right|<1 .
$$

When the inequalities (39) are broken down the functions $\Phi_{z}(\eta ; u, v, w)$ still are solutions of eq. (35) and could be considered as non normalizable eigenstates. In case of $u=0$ in eq. (35) we have (in view of (36) first order equation to solve 31]. It turned out that the solutions for this case could be obtained from $\Phi_{z}(\eta ; u, v, w ; k)$ taking appropriate limits in it. One can check that the conditions (39) can be satisfied by real $w$ and $v=u^{*}$ when the operator $u K_{-}+v K_{+}+w K_{3}$ becomes hermitean. Then the algebraic states $\left|z, u, u^{*}, w\right\rangle\left(w=w^{*}\right)$ are RIS for the three observables $K_{1}, K_{2}$ and $K_{3}$. RIS for the nonsquare integrable representations corresponding to $k=1 / 2,3 / 4$ are considered in next subsection.

Various known states are contained in the large family of $s u(1,1)$ states $|z, u, v, w ; k\rangle$ [31]. In particular, when $w=0$ we get the SIS $|z, u, v ; k\rangle$ for the noncompact generators $K_{1}$ and $K_{2}$, which in turn at $z=-k \sqrt{-u v}$ 15 recover the family of $S U(1,1)$ group CS $|\tau ; k\rangle$ (the squeezed vacuum states [3]), $\tau=\sqrt{-v / u},|\tau|<1$. In view 
of the positivity of the commutator $i\left[K_{1}, K_{2}\right]=\left[K_{-}, K_{+}\right] / 2$ the uncertainty matrix $\sigma\left(K_{1}, K_{2} ; \rho\right)$ is positive definite and therefor possesses the resulting properties, described in section 2. In IS $|z, u, v ; k\rangle$ the matrix elements of $\sigma$ are

$$
\sigma_{11}=\frac{1}{2} \frac{|u-v|^{2}}{|u|^{2}-|v|^{2}}\left\langle K_{3}\right\rangle, \quad \sigma_{22}=\frac{1}{2} \frac{|u+v|^{2}}{|u|^{2}-|v|^{2}}\left\langle K_{3}\right\rangle, \quad \sigma_{12}=\frac{\operatorname{Im}\left(u^{*} v\right)}{|u|^{2}-|v|^{2}}\left\langle K_{3}\right\rangle,
$$

satisfying $\operatorname{det} \sigma=\operatorname{det} C=\left\langle K_{3}\right\rangle^{2} / 4$. The $K_{1}-K_{3}$ and $K_{2}-K_{3}$ IS are obtained from $|z, u, v, w ; k\rangle$ when $v=u$ and $v=-u$ respectively.

The case of $s u(2)$ RIS (i.e. spin RIS) can be treated in a similar manner using the representation of $S U(2)$ group related CS $|\zeta ; j\rangle$ in which [3]

$$
J_{-}=-\zeta^{2} \frac{d}{d \zeta}+2 j \zeta, \quad J_{-}=\frac{d}{d \zeta}, \quad J_{3}=\zeta \frac{d}{d \zeta}-j .
$$

Here $j=1 / 2,1,3 / 2, \ldots, \quad\left[J_{-}, J_{+}\right]=-2 J_{3},\left[J_{3}, J_{ \pm}\right]= \pm J_{ \pm} \quad\left(J_{ \pm}=J_{1} \pm i J_{2}\right)$ and $J^{2}=J_{1}^{2}+J_{2}^{2}+J_{3}^{2}=j(j+1)$. The required eigenvalue problem

$$
\left(\beta_{\nu} J_{\nu}\right)|z, \vec{\beta} ; j\rangle=z|z, \vec{\beta} ; j\rangle
$$

where $\vec{\beta}=\left(\beta_{1}, \beta_{2}, \beta_{3}\right),\left(\beta_{\nu}\right.$ are complex parameters) is solved by Brif [30. In case

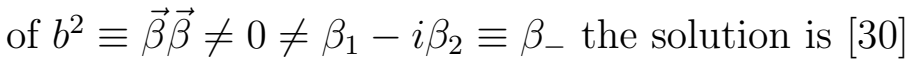

$$
\Phi_{z}(\zeta ; \vec{\beta}, j)=N_{0}\left(\zeta-\frac{\beta_{3}-b}{\beta_{-}}\right)^{j+z / b}\left(\zeta-\frac{\beta_{3}+b}{\beta_{-}}\right)^{j-z / b}
$$

with the normalizability condition $z=m b, m=-j,-j+1, \ldots, j-1, j$. As we expect these $s u(2)$ RIS contain the set of standard $S U(2)$ CS with maximal symmetry $\left|\zeta^{\prime} ; j\right\rangle$ and this occurs when $m= \pm j$ with $\zeta^{\prime}=-\beta_{-}\left(\zeta_{3} \mp b\right)^{-1}$ [30. At $\beta_{3}=0$ the $s u(2)$ RIS coincide with the Schrödinger $J_{1^{-}} J_{2}$ IS considered in ref. 15.

For the $s u(2)$ observables $J_{\nu}$ (the spin components) it is important to note that the spin component uncertainty matrix $\sigma(\vec{J} ; \rho)$ in any state can be diagonalized by means of orthogonal linear transformation of $J_{\nu}$. The latter can be induced by rotation of coordinates in $\mathrm{R}_{3}$ since $s u(2) \sim s o(3)$. Therefor we deduce that spin component correlations are of pure coordinate nature - they can be eliminated in any state by rotations of the reference frame. Here one can also perform second kind diagonalization of $\sigma$, keeping $J_{\nu}$ and transforming the state $\rho$ by an unitary operator $U(g)$ of $S U(2) \sim S O(3)$. Thus correlated spin RIS are unitary equivalent to noncorrelated spin RIS.

\subsection{RIS of multimode boson systems}

In this subsection we first consider $n=2 N$ canonical operators $p_{j}$ and $q_{j}, j=$ $1,2, \ldots, N$, which are quadrature components of $N$ boson/photon destruction (creation) operators $a_{j}=\left(q_{j}+i p_{j}\right) / \sqrt{2}\left(a_{j}^{\dagger}=\left(q_{j}-i p_{j}\right) / \sqrt{2}\right):\left[q_{j}, p_{k}\right]=i \delta_{j k}$. Here we 
put for concreteness $X_{\nu} \equiv Q_{\nu}, Q_{j}=p_{j}, Q_{N+j}=q_{j}$ and $A_{j}=q_{j}+i p_{j}=a_{j} \sqrt{2}$. The set of $Q_{\mu}$ and the unity operator close the Heisenberg algebra $h_{N}$, which is nilpotent (therefor non semisimple). So RIS for canonical observables $Q_{\mu}$ are $h_{N}$ RIS (to be called also multimode amplitude RIS). According to the proposition 3 eigenstates $|\vec{\alpha}, u, v\rangle \equiv|\vec{\alpha}, \Lambda\rangle, \quad \vec{\alpha}=\left(\alpha_{1}, \alpha_{2}, \ldots, \alpha_{N}\right)$, of $a_{j}^{\prime}$,

$$
a_{j}^{\prime}=u_{j k} a_{k}+v_{j k} a_{j k}^{\dagger}=\frac{1}{2}\left[\left(\lambda_{q}\right)_{j k} q_{k}+\left(\lambda_{p}\right)_{j k} p_{k}\right],
$$

with any $u$ and $v$ are RIS for $Q_{\mu}$,

$$
a_{j}^{\prime}|\vec{\alpha} ; u, v\rangle=\alpha_{j}|\vec{\alpha} ; u, v\rangle, \quad j=1,2, \ldots, N
$$

Here $u=\left(\lambda_{q}-i \lambda_{p}\right) / 2, v=\left(\lambda_{q}+i \lambda_{p}\right) / 2$ and $u, v, \lambda_{q}$ and $\lambda_{p}$ are $N \times N$ complex matrices. The $N \times N$ matrices $\lambda_{q}$ and $\lambda_{p}$ are related to the transformation matrix $\Lambda$ in (司) (which now is rewritten as $Q_{\mu}^{\prime}=\lambda_{\mu \nu} Q_{\nu}$ ) as follows:

$$
\Lambda=\left(\begin{array}{cc}
\lambda_{1} & \lambda_{2} \\
\lambda_{3} & \lambda_{4}
\end{array}\right), \quad \lambda_{p}=\lambda_{3}+i \lambda_{1}, \lambda_{q}=i \lambda_{2}+\lambda_{4}
$$

If one impose the symplectic conditions $\Lambda J \Lambda^{T}=J$ on $\Lambda$ the operators $a_{j}^{\prime}$ become new annihilation operators, i.e. the linear transformation (5) becomes canonical one. With this conditions $\vec{Q}$-RIS are unitary equivalent (with methaplectic operator $U(\Lambda)$ ) to eigenstates of $a_{j}$, i.e. to canonical multimode CS $|\vec{\alpha}\rangle$. In ref. [11] states $|\vec{\alpha}, \Lambda(t)\rangle$ were constructed explicitly as solution $|\vec{\alpha} ; t\rangle$ of time dependent Schrödinger equation for general quadratic, possibly time dependent, Hamiltonian $H=B_{\mu \nu}(t) Q_{\mu} Q_{\nu}$ (plus linear terms as well). In terms of parameter matrices $\lambda_{q}$ and $\lambda_{p}$ these canonical RIS $|\vec{\alpha}, \Lambda\rangle=|\vec{\alpha}, u, v\rangle$ in coordinate representation read $(\Delta=0$ in eqs. (17) of third paper of ref. [11])

$$
\langle\vec{q} \mid \vec{\alpha} ; \Lambda\rangle=\pi^{N / 4} \exp \left(\gamma+\overrightarrow{\tilde{\nu}} \vec{q}-\frac{1}{2} \vec{q} \tilde{\mu} \vec{q}\right),
$$

where $\tilde{\mu}$ is $N \times N$ matrix, $\tilde{\mu}=i \lambda_{p}^{-1} \lambda_{q}, \overrightarrow{\tilde{\nu}}$ is $N$ vector, $\overrightarrow{\tilde{\nu}}=(1 / \sqrt{2})\left(\lambda_{q}^{\dagger}-\lambda_{p}^{-1} \lambda_{q} \lambda_{p}^{\dagger}\right) \vec{\alpha}$ and

$$
\gamma=-\frac{1}{2}|\vec{\alpha}|^{2}+\frac{i}{4} \vec{\alpha}\left(\lambda_{p}^{*} \lambda_{p}^{-1} \lambda_{q} \lambda_{p}^{\dagger}-\lambda_{q}^{*} \lambda_{p}^{\dagger}\right) \vec{\alpha}
$$

At $\Lambda=1$ the RIS (47) coincide with canonical CS $|\vec{\alpha}\rangle$ in coordinate representation. The multimode states (47) in deferent parametrizations were also considered in several papers under the names multimode squeezed states [33] or multimode/polymode correlated states [19, 34, 35] or Gaussian pure states [35].

It is worth noting that for canonical RIS the condition $(45) \sim(19)$ is not only sufficient, but also necessary, i.e. all $\vec{Q}$-RIS are eigenstates of $a_{j}^{\prime}=u_{j k} a_{k}+v_{j k} a_{j k}^{\dagger}$ for some $u_{j k}$ and $v_{j k}$. This can be proved using the diagonalization of $\sigma(\vec{Q}, \rho)$. 
Indeed, let $\sigma^{\prime}=\sigma\left(\overrightarrow{Q^{\prime}}, \rho\right)=\sigma\left(\vec{Q}, \rho^{\prime}\right)$ be diagonal. Here $\rho^{\prime}=U(\Lambda) \rho U^{\dagger}(\Lambda)$, where $U$ is methaplectic unitary operator. Since the mean commutator matrix $C$ now is constant, $\operatorname{det} C=4^{-N}$ the equality in RUR is $\operatorname{det} \sigma=\operatorname{det} \sigma^{\prime}=\prod_{j}^{N} \sigma_{j j}^{\prime} \sigma_{N+j, N+j}^{\prime}=$ $4^{-N}$. Since for every $j$ the product $\sigma_{j j}^{\prime} \sigma_{N+j, N+j}^{\prime}$ is greater or equal to $1 / 4$ we get that all products should be equal to $1 / 4$. But this is possible if and only if $\rho$ is pure multimode CS for new variables $\overrightarrow{Q^{\prime}}$, that is $\rho$ is pure state, methaplectically equivalent to multimode CS for old variables $\vec{Q}, \rho=U(\Lambda)|\vec{\alpha}\rangle\langle\vec{\alpha}| U^{\dagger}(\Lambda)$.

Consider briefly now the uncertainty matrix of canonical observables $\sigma(\vec{Q}, \rho)$. Since $Q_{\mu}$ satisfy the requirements of proposition 2 the $\sigma(\vec{Q}, \rho)$ is positive definite. Therefor it can be diagonalized by means of linear canonical transformation in any state $\rho$ and it obey the inequalities (16). In $\vec{Q}$-RIS $|\vec{\alpha}, \Lambda\rangle$ the dispersion matrix $\sigma(\vec{Q} ; \vec{\alpha}, \Lambda)$ has further properties. The main one is that $\sigma(\vec{Q} ; \vec{\alpha}, \Lambda)$ is symplectic itself. Indeed we have

$$
\sigma(\vec{Q} ; \vec{\alpha}, \Lambda)=\sigma\left(\overrightarrow{Q^{\prime}} ; \vec{\alpha}, 1\right)=\Lambda \sigma(\vec{Q} ; \vec{\alpha}, 1) \Lambda^{T}
$$

where $\sigma(\vec{Q} ; \vec{\alpha}, 1)$ is the uncertainty matrix in multimode canonical CS $|\vec{\alpha}\rangle$. The latter is evidently proportional to the unity, $\sigma(\vec{Q} ; \vec{\alpha}, 1)=\frac{1}{2}$ and therefor if $\Lambda$ is symplectic then $2 \sigma(\vec{Q} ; \vec{\alpha}, \Lambda)$ is also symplectic. We express $\sigma$ in terms of $N \times N$ uncertainty matrices $\sigma_{p p}, \sigma_{q q}, \sigma_{q p}$ and $\sigma_{p q}=\sigma_{q p}^{T}$

$$
\sigma(\vec{Q})=\left(\begin{array}{ll}
\sigma_{p p} & \sigma_{p q} \\
\sigma_{q p} & \sigma_{q q}
\end{array}\right)
$$

and write the symplectic properties of $\sigma(\vec{Q} ; \vec{\alpha}, \Lambda)$ in $N \times N$ matrix form,

$$
\sigma_{p p} \sigma_{q p}-\sigma_{p g} \sigma_{p p}=0=\sigma_{q p} \sigma_{q q}-\sigma_{q q} \sigma_{p q}, \quad \sigma_{p p} \sigma_{q q}-\sigma_{p q}^{2}=1 / 4
$$

For $N=1$ the last equality is just the equality in Schrödinger relation (3), the first two being satisfied identically in any state.

For boson systems it is of interest to consider observables which are quadratic combinations of creation and annihilation operators $a_{j}^{\dagger}$ and $a_{k}$ (or equivalently of $p_{j}$ and $q_{k}$ ). Quadratic combinations

$$
K_{j k}=\frac{1}{2} a_{j} a_{k}, \quad K_{j k}^{\dagger}=\frac{1}{2} a_{k}^{\dagger} a_{j}^{\dagger}, \quad K_{j k}^{(3)}=\frac{1}{4}\left(a_{j}^{\dagger} a_{k}+a_{k}^{\dagger} a_{j}\right)
$$

close the simple noncompact algebra $\operatorname{sp}(N, R)$ [6], the noncompact elements being spanned by lowering and raising operators $K_{j k}$ and $K_{j k}^{\dagger}$. In the one mode case $s p(1, R) \sim s u(1,1)$ and

$$
\frac{1}{2} a^{2}=K_{-}, \quad \frac{1}{2} a^{\dagger 2}=K_{+}, \quad \frac{1}{4}\left(2 a^{\dagger 2} a+1\right)=K_{3} .
$$


Operators (51) are generators of the methapletic group $M p(N, R)$, which covers the $S p(N, R) . s p(N, R)$ RIS in the representation (51) should be called multimode squared amplitude RIS. RIS for the quadratures $X_{j k}$ and $Y_{j k}$ of $K_{j k}, K_{j k}=X_{j k}+i Y_{j k}$ (shortly $K_{j k}$-RIS), are eigenstates of $N \times N$ complex combinations of lowering and raising operators $K_{j k}$ and $K_{j k}^{\dagger}$ and according to our general result they contain group related $M p(N, R)$ CS with maximal symmetry, $|\psi(g)\rangle=U(g)|0\rangle, U(g) \in M p(N, R)$, the exstremal vector being the multimode boson vacuum $|\overrightarrow{0}\rangle$ (these CS coincide with multimode squeezed vacuum states [19, 33, 34]). On the other hand $M p(N, R)$ CS are annihilated by all $a_{j}^{\prime}=U(g) a_{j} U^{-1}(g)=u_{j k} a_{k}+v_{j k} a_{k}^{\dagger}$. Herefrom we get the property that $M p(N, R)$ CS with maximal symmetry are simultaneously $h_{N}$ and $s p(N, R)$ RIS (i.e. amplitude and squared amplitude multimode RIS, double IS). In coordinate representation and in parametrization by $\lambda_{q}$ and $\lambda_{p}\left(\vec{a}^{\prime}=\lambda_{q} \vec{q}+\lambda_{p} \vec{p}\right)$ these multimode double IS are given by formula (47) with $\vec{\alpha}=0$.

An other explicit example of $s p(N, R)$ RIS is given by multimode squeezed Fock states $U(g)|\vec{n}\rangle$, where $U(g) \in M p(N, R)$. Indeed, Fock states $|\vec{n}\rangle$ are eigenstates of hermitean $M p(N, R)$ generators $K_{j j}^{(3)}=a_{j}^{\dagger} a_{j} / 2$ (see eq. (51)), therefor $U(g)|\vec{n}\rangle$ are eigenstates of hermitean operators $U(g) K_{j j}^{(3)} U(g)^{\dagger}$ which are real linear combinations of all $M p(N, R)$ generators (follows from the $\mathrm{BCH}$ formula). From section 3 we know that this eigenvalue property is sufficient for the equalities $\operatorname{det} \sigma=\operatorname{det} C=0$, i.e. the squeezed Fock states are $s p(N, R)$ RIS for all hermitean quadratures of operators (51). Multimode squeezed Fock states $U(g)|\vec{n}\rangle$ were constructed in the last two papers of ref. [11], where the $M p(N, R)$ operator $U(g)$ was taken as evolution operator $U(t)$ of general $N$ dimensional quadratic quantum system (in coordinate representation the states $\langle\vec{q}|U(g)| \vec{n}\rangle$ were expressed as product of $\langle\vec{q} \mid \overrightarrow{0}\rangle$ (see eq. (47)) and a Hermite polynomial of $N$ variables). Note that squeezed Fock states are $\operatorname{sp}(N, R)$ RIS and not $h_{N}$ RIS and squeezed Glauber CS are $h_{N}$ RIS and not $s p(N, R)$ RIS. Only squeezed vacuum states are simultaneously $s p(N, R)$ RIS and $h_{N} \operatorname{RIS}\left(h_{N} \operatorname{RIS}=\vec{Q}\right.$-RIS).

Recently attention is paid in the physical literature to multimode even and odd CS [36] $|\vec{\alpha}\rangle_{ \pm}=N_{ \pm}(|\vec{\alpha}\rangle \pm|-\vec{\alpha}\rangle)$, where $|\vec{\alpha}\rangle=D(\vec{\alpha})|\overrightarrow{0}\rangle$ is Glauber multimode CS. We readily see that these $|\vec{\alpha}\rangle_{ \pm}$are eigenstates of all $K_{j k}$, eq. (51), and therefor are noncorrelated squared amplitude RIS with equal uncertainties of quadratures of $K_{j k}$. It is the set of all $s p(N, R) K_{j k}$-RIS which is a natural extension of that of multimode even and odd CS $|\vec{\alpha}\rangle_{ \pm}$, incorporating also the multimode squeezed vacuum states $|\overrightarrow{0}, u, v\rangle$ and Glauber CS $|\vec{\alpha}\rangle$. Unlike the even and odd CS $|\vec{\alpha}\rangle_{ \pm}$the $K_{j k}$-RIS (being eigenstates of combinations $u_{j k} a_{j} a_{k}+v_{j k} a_{j}^{\dagger} a_{k}^{\dagger}$ ) can exhibit strong squeezing in quadratures of $a_{j} a_{k}$ and therefor can be called multimode squared amplitude squeezed states in complete analogy to the well known case of multimode 
(amplitude) squeezed states [19, 33, 34.

We underline that the set of all $\operatorname{sp}(N, R)$ RIS, and even the set of the $K_{j k}$-RIS is much larger than the set of $M p(N, R)$ CS $U(g)|0\rangle$. The problem can be solved entirely in the one mode case, $N=1$, using Glauber CS representation, in which $a=d / d \alpha, a^{\dagger}=\alpha$ [30, 31]. The resulting even states take the form (37) with the replacements $k=1 / 4$ and $\eta=\alpha^{2} / 2$, the normalizability conditions remaining the same as (39). Some particular sets of one mode squared amplitude squeezed states are constructed and discussed in [17]. Generalized one mode even and odd CS $|z, u, v ; \pm\rangle$ were first constructed in the second paper of ref. [19] as even and odd solutions of the eigenvalue equation $\left(u a^{2}+v a^{\dagger 2}\right)|z, u, v ; \pm\rangle=z|z, u, v ; \pm\rangle$ with complex parameters $u$ and $v$. Eigenvalue problem for operators $\left(a+\zeta a^{\dagger}\right)^{2}(\zeta \in \mathbb{C}$ was considered in [37.

The RIS which are not group related CS exhibit many physical properties which group CS lack. One of such properties is squeezing in fluctuation of group generators. Squeezing in fluctuation of $X_{\mu}$ in a state $|\psi\rangle$ occurs if $|\psi\rangle$ is close (by norm form example) to an eigenstate of $X_{\mu}$ since the (squared) variance $\Delta^{2} X_{\mu}=\sigma_{\mu \mu}$ of $X_{\mu}$ vanishes in eigenstates of $X_{\mu}$ only [15]. Therefor if in RIS which is eigenstate of $\beta_{\nu} X_{\nu}$ all but $\beta_{\mu}$ tend to 0 then $\Delta X_{\mu}$ should tend to 0 . In group CS with symmetry it is not always possible to let all but $\beta_{\mu}$ to tend to 0 due to constrain (31) (it is trivially possible if $X_{\mu}$ itself has $\left|\psi_{0}\right\rangle$ as its eigenstate). In the case of $s u(1,1)$ we have explicit solutions $|z, u, v, w ; k\rangle$, eq. (37) and $\mathrm{CS}|\tau ; k\rangle$ and one can verify the above statement: the variances of $K_{1,2}$ in CS are grater than $k$ for any $\tau$ [15], while for example for $k=1 / 4$ the $K_{1}-K_{2}$ IS $|z, u, v ; 1 / 4\rangle$ with $z=-1, u=\sqrt{1+x^{2}}, v=-x<0$ exhibit strong squeezing in $K_{2}\left(\Delta K_{2}\right.$ is monotonically decreasing when $x$ increases). Moreover one can find IS which exhibit $K_{1}\left(K_{2}\right)$ and $q(p)$ squeezing (joint amplitude and squared amplitude squeezing) simultaneously. Subpoissonian statistics also occurs in IS $|z, u, v, w ; 1 / 4\rangle$. In greater detail nonclassical properties of $S U(1,1)$ IS (for $k=1 / 4,3 / 4$ ) are discussed (and illustrated by several graphics) in 31.

By means of 4 boson operators $a, b, a^{\dagger}, b^{\dagger}$ one can construct quadratic combinations which close $s u(1,1)$ (the representations with Bargman index $k=(1+$ $\left.\left|n_{a}-n_{b}\right|\right) / 2=1 / 2,1, \ldots$, considered in the previous subsection) or $s u(2)$ algebra (the Schwinger realization), which are subalgebras of $\operatorname{sp}(4, R)$, eq. (51) for $N=2$. Currently physical properties of various $s u(1,1)$ and $s u(2)$ SIS of two mode boson/photon system are being discussed (see [38, 39, 40] and references therein). We note the result of 40]: $K_{2}-K_{3}$ two mode IS which are not $S U(1,1)$ group CS can improve the sensitivity in the interferometric measurements. Several schemes of generation of SIS for $s u(1,1)$ or $s u(2)$ operators in two mode quadratic boson representations are considered recently [38, 39, 40]. But so far no scheme of generation 
of $K_{1}-K_{2}$ one mode SIS is presented. It seems natural to generate these SIS from experimentally available Glauber CS or even and odd CS [8] acting on the laster by the squared amplitude squeeze operator $S(u, v)$, eq. (27). For this purpose however one has to look for a nonunitary evolution process, since here the squeeze operator $S(u, v)$ is isometric only [31.

\section{Concluding remarks}

We have shown that the uncertainty matrix for $n$ observables $X_{\mu}$ can always be diagonalized by linear transformation of $X_{\mu}$. For the case of spin component operators this means that spin covariances are of pure coordinate origin and correlated spin states are unitary equivalent to noncorrelated states. When the uncertainty matrix is positive definite (as is the case e.g. of $q$-deformed multimode boson system with $q>0$, in particular the case of canonical boson system, $q=1$ ) it can be diagonalized by means of symplectic transformations. Using the above diagonalization property a new family of uncertanty relations for positive definite uncertainty matrices is established.

The Robertson $n$ dimensional relation for the uncertainty matrix, eq. (1), is shown to be efficient in generalization of the basic properties of Glauber coherent states (CS) to arbitrary system of observables $X_{\mu}$. For even number $n$ of observables this relation is minimized in a state $|\psi\rangle$ if $|\psi\rangle$ is eigenstate of $n / 2$ independent complex combinations of $X_{\mu}$. For any (even or odd) $n$ the minimization occurs in states which are eigenvectors of real combination of $X_{\mu}$. When $X_{\mu}$ close a semisimple Lie algebra the set of states which minimize the Robertson inequality (called here Robertson intelligent states (RIS)) contain the corresponding group related CS with symmetry. CS with maximal symmetry are contained also in RIS for the quadratures of Weyl lowering and raising operators. Thus it is the Robertson uncertainty relation that brings together the three ways of generalization of Glauber CS [3] to the level of $n$ observables.

RIS which are not group related CS can exhibit interesting physical properties. One such universal property to be distinguished from CS is the strong squeezing of group generators. In this way the multimode squared amplitude squeezed states are naturally introduced as $s p(N, R)$ RIS. Squared amplitude RIS can exhibit both linear and quadratic squeezing as we have shown on the example of $K_{1}-K_{2}$ IS. Such joint squeezing of noncommuting observables could be useful in optical communications and interferometric measurements since the field in such squeezed states is better determined - this should be considered elsewhere. The problem of generation of RIS for two $s u(1,1)$ and $s u(2)$ observables is discussed in recent papers [38, 39, 40]. 
In this connection we note the principle possibility to generate e.g. $K_{1}-K_{2}$ squared amplitude IS by means of isometric (non unitary) evolution operators.

Acknowledgement. The work is partially supported by Bulgarian Science Foundation, Contracts No. F-559.

\section{References}

[1] Glauber R J 1963, Phys. Rev. 1311726

Klauder J R 1963, J. Math. Phys. 41005.

[2] Klauder J R and Skagerstam B S 1985. Coherent States (W. Scientific, Singapore, 1985).

[3] Zhang W M, Feng D H and Gilmore R 1990, Rev. Mod. Phys. 62, 867.

[4] Robertson H R 1934, Phys. Rev. 46794.

[5] Dodonov V V and Man'ko V I 1987, in Trudy FIAN, v. 183, p.1-70 ("Nauka", Moskva, 1987) (Nuova Science, Commack, N.Y., 1988).

[6] Barut A O and Raszcka R 1977. Theory of Group Representations and Applications (Polish Publishers, Warszawa, 1977).

[7] Aragone C, Chalband E and Salamo S 1976, J. Math. Phys. 171963.

[8] Dodonov V V, Kurmyshev E and Man'ko V I 1980, Phys. Lett. A79 150.

[9] Malkin I A, Man'ko V I and Trifonov D A 1969, Phys. Lett. A30 413

[10] Malkin I A, Man'ko V I and Trifonov D A 1970 Phys. Rev. D2 1371

Stoler D A 1970, Phys. Rev. D1 3217

Lu E Y C 1971, Lett. N. Cimento 2, 1241.

[11] Holz A 1970, Lett. N. Cimento A4 1319

Malkin I A, Man'ko V I and Trifonov D A 1971, N. Cimento A4 773

- 1973, J. Math. Phys. 14576.

[12] Schrödinger E 1930, in Sitz. der Preuss. Acad. Wiss. (Phys.-Math. Klasse, p.296) (Berlin, 1930).

[13] Trifonov D A 1993, J. Math. Phys. 34100.

[14] Loudon R and Knight P 1987, J. Mod. Opt. 34, 709

Walls D F 1983, Nature 306141.

[15] Trifonov D A 1994a, J. Math. Phys. 35, 2297

- 1994b, Phys. Lett. A187 284.

[16] Barut A O and Girardello L 1971, Commun. Math. Phys. 2141. 
[17] Hillery M 1984, Phys. Rev. A36 3796

Bergou J A, Hillery M and Yu D 1991, Phys. Rev. A43, 515

Nieto M M and Truax D R, Phys. Rev. 1993, Lett. 712843

Gerry C C and Grobe R 1995, Phys. Rev. 514123

Puri R R and Agarwal G S 1996, Phys. Rev.A53(3), 1786.

[18] Provost J and Vallée G 1980, Commum. Math. Phys. 76289

Nikolov B A and Trifonov D A 1988, Bulg. J. Phys. 15, 33

Abe S 1993, Phys. Rev. A48, 4102

Spera M 1993, J. Geom. Phys. 12, 165.

[19] Sudarshan E C G, Chiu C B and Bhamathi G 1995, Phys. Rev. A52, 43

Trifonov D A 1995, Preprint INRNE-TH-95/5.

[20] Gantmaher F R 1975. Teoria Matrits (Moskva, "Nauka", 1975).

[21] Colpa J H 1978, Physica 93A, 327

Bogdanovic R and Gopinathan M 1979, J. Phys. A12 1457.

[22] Seleznyova A N 1995, Phys. Rev. A51 950.

[23] Macfarlane A J 1989, J. Phys. A22 4581

Biedenharn L C 1989, J. Phys. A22 L873.

[24] Oh C H and Singh K 1994, J. Phys. A27 5907.

[25] Kuang L M and Wang F S 1993, Phys. Lett. A173 221.

[26] Aldaya V and Guerrero J 1995, J. Math. Phys. 363191.

[27] Agarwal G S 1988, J. Opt. Soc. Am. B5 1940

Sun J, Wang J and Wang C 1991, Phys. Rev. A44 3369

Hach III E E and Gerry C C 1992, J. Mod. Opt. 392501.

[28] Epifanio G, Todorov T C and Trapani S 1996, J. Math. Phys. 371148.

[29] Naimark M A 1976. Theory of Group representations ("Nauka", Moskva, 1976).

[30] Brif C 1996, Ann. of Phys. 251180

- 1997 E-print quant-ph/9701003.

[31] Trifonov D A 1996, E-prints quant-ph/9609001, quant-ph/9609017.

[32] Handbook of mathematical functions, edited by M. Abramowitz and I A Stegun (National bureau of standards, 1964) (Russian translation, M. "Nauka", 1979).

[33] Ma X and Rhodes W 1990, Phys. Rev. 414624.

[34] Dodonov V V, Man'ko O V and Man'ko V I 1994, Phys. Rev. A50 813 Man'ko V I 1996, E-print quant-ph/9601023 (also in NASA Conference Publication 3322, Greenbelt, MD, 1996) p. 115). 
[35] Sudarshan E C G 1993, in NASA Conference Publication No. 3219 (NASA, Greenbelt, MD, 1993), p. 241

Simon R, Sudarshan E C G and Mukunda N 1988, Phys. Rev. A36 3868.

[36] Man'ko Olga 1996, Preprint ICTP (Trieste) IC/96/39

Dodonov V V, Man'ko V I and Nikonov D E 1995, Phys. Rev. A51 3328

Ansari N A and Man'ko V I 1994, Phys. Rev. A50 1942.

[37] A. Wünsche 1995, Acta Phys. Slovaca 45413.

[38] Luis A and Perina J 1996, Phys. Rev. A53 1886.

[39] Brif C and Ben-Aryeh Y 1996, Quantum Semiclass. Opt. 81.

[40] Brif C and Mann A 1996, Phys. Rev. A54 4505. 